\title{
Angle Displacement Robust Controller for the Port Plate of the Hydraulic Transformer
}

\author{
Wei Shen, ${ }^{1}$ Jihai Jiang, ${ }^{1}$ Xiaoyu Su, ${ }^{2}$ and Hamid Reza Karimi ${ }^{3}$ \\ ${ }^{1}$ School of Mechatronics Engineering, Harbin Institute of Technology, Harbin 150080, China \\ ${ }^{2}$ College of Automation, Harbin Engineering University, Harbin 150001, China \\ ${ }^{3}$ Department of Engineering, Faculty of Technology and Science, University of Agder, 4898 Grimstad, Norway
}

Correspondence should be addressed to Wei Shen; shenw@hit.edu.cn

Received 21 August 2013; Accepted 15 October 2013

Academic Editor: Tao Li

Copyright (c) 2013 Wei Shen et al. This is an open access article distributed under the Creative Commons Attribution License, which permits unrestricted use, distribution, and reproduction in any medium, provided the original work is properly cited.

\begin{abstract}
The hydraulic transformer is used in the hydraulic system to enhance the efficiency. However, how to control the angle displacement of the port plate is becoming a critical issue because of the new structure of the hydraulic transformer. This paper presents a new method for the angle displacement control system. Firstly, the basic principle of the system is presented. Then, the disturbance which is mainly the friction torque between the port plate and the cylinder block is calculated to estimate the range. Furthermore, the guaranteed cost control (GCC) is analyzed and combined with the disturbance and the characteristics of parameter uncertainties. Finally, the proposed control method is compared with the traditional PI control and the simulation result shows the effectiveness of the proposed design method.
\end{abstract}

\section{Introduction}

Energy saving research in the field of hydraulic system is becoming a hot point [1-6]. Common pressure rail (CPR) is one promising hydraulic architecture because of its many advantages, for instance, not only that it eliminates the throttling loss in the theoretical aspect, but also has the module characteristic $[7,8]$. In $\mathrm{CPR}$, the constant pressure variable pump and hydraulic accumulator constitute the high pressure oil sources, and multiple different loads connect in parallel between the high pressure and the low pressure oil pipe [912]. Basically, there are two kinds of actuators in CPR. They are hydraulic variable displacement motors and cylinders. For the rotating load, the control target such as position, velocity, or power can be reached by regulating the displacement of hydraulic pump/motor. However, because it is hard to change the displacement of the hydraulic cylinder, hydraulic transformer (HT) is introduced to control the hydraulic cylinder for adapting to load change without throttling loss from the theoretical aspect. The detailed information about HT can be found in [13]. Hence, HT is used to control the hydraulic cylinder by regulating the angle of the port plate. However, because the structure of the port plate is different from the traditional axial piston type component, how to control the angle of port plate is a new challenge. For the traditional axial piston pump, the port plate is fixed with the case and it cannot be moved. Hence, the force applied on the swash plate from the cylinder block and some other components of the pump is balanced by the case. However, the torque applied on the port plate of HT should be considered because of the rotation motion during controlling its angle. Figure 1 shows the structure of the HT [14]. The external load torque, which is mainly the friction torque between the cylinder block and the port plate, is also changing during the regulating process. This can be explained by introducing the working principle of HT.

Figure 1(b) shows the structure of the port plate which has three ports [13]. They are connected with the high pressure pipe of CPR, the load, and the low pressure pipe of CPR, respectively. The cylinder block of the HT is driven by the sum of torque generated by the three ports of the SHT. The sum of torque among three ports is

$$
\Delta T=J_{\mathrm{HT}} \dot{\omega}_{\mathrm{HT}}=T_{A}+T_{B}+T_{T},
$$

where $J_{\mathrm{HT}}$ is the moment of inertia of HT and $\omega_{\mathrm{HT}}$ is the angular speed of the cylinder block of HT. 


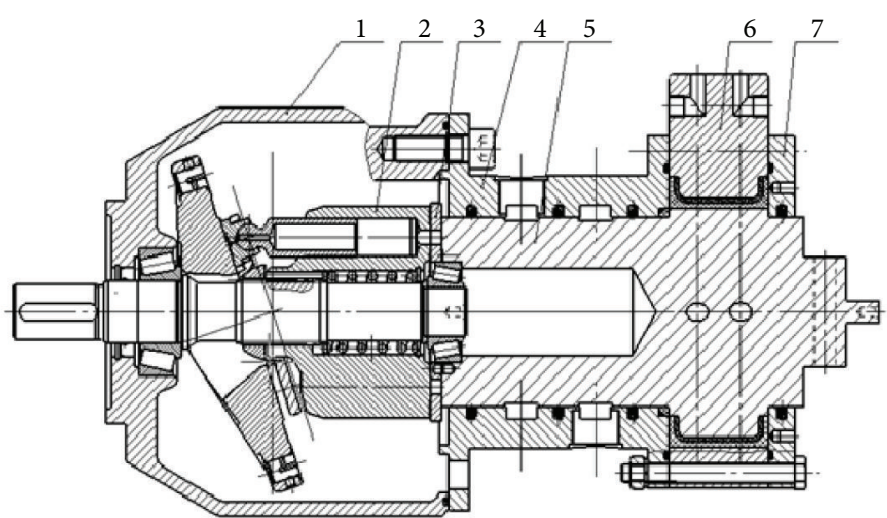

(a)

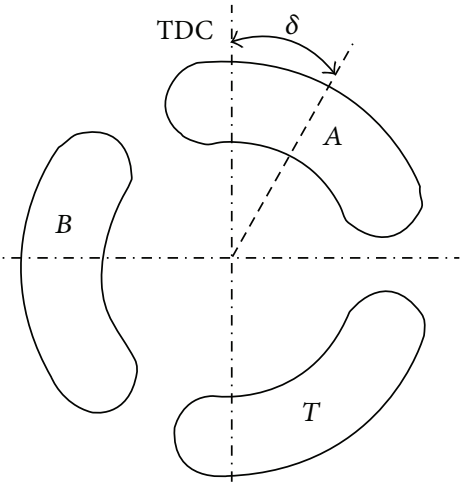

(b)

Figure 1: Structure of HT. (1) Case of original pump. (2) Cylinder block. (3) New port plate. (4) Case of swing motor. (5) Rotor of swing motor. (6) Stator of swing motor. (7) Rear cover of swing motor.

The torque applied on the port plate should be changing because of the changing load and then the difficulty for a good control performance is increased. Until now, there are already many papers which are focusing on the robust control [15-21]; for instance, in [22], the authors deal with the robust control problem with parametric uncertainties for delayed singular systems. In [23], the paper describes robust force sensorless control system in motion control. In addition, the robust control theory is applied on diesel engine selective catalytic reduction (SCR) systems to enhance the performance [24]. Because the guaranteed cost control (GCC) not only stabilizes the uncertain system robustly but also ensures an adequate level of performance, in which an upper bound on the closedloop value of a quadratic cost function can be guaranteed by using a fixed Lyapunov function [25], the GCC becomes an effective method for enhancing the system performance [2630]. However, there is no method that focuses on the angle displacement control of the port plate. In general, the critical problems can be described by how to resist the interference which was applied on the port plate and eliminated the influence of the uncertain model. In this paper, the GCC is introduced to the field of the angle displacement control for the port plate. Firstly, the mathematical model of the angle displacement system is constructed. Secondly, the range of the disturbance and the uncertain parameter must be estimated in order to apply the GCC theory. Moreover, the simulation about the GCC control and the PI control is conducted by using Matlab. Finally, the simulation result is explained.

\section{Modeling}

The basic schematic is shown in Figure 2. The main components of the system are (1) pump and (2) servovalve, and both (3-1) swing motor and (3-2) HT main part are one piece in practical applications. However, this paper is focused on the valve controlling the motor, and the information about other parts can be found in [31].

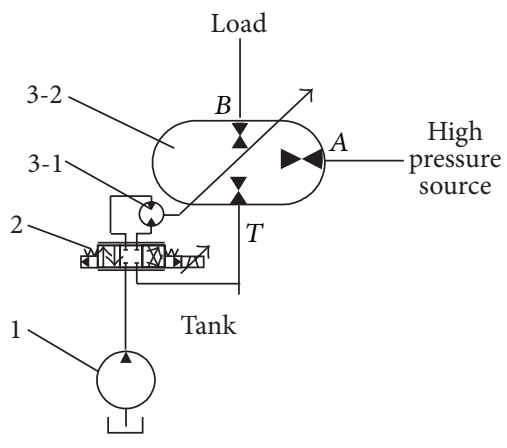

Figure 2: Hydraulic schematic of the system.

2.1. Servovalve Flow Rate Equation. The linearized servovalve flow equation is

$$
Q_{L}=K_{q} K_{i} K_{s} u_{i}-K_{c} p,
$$

where $Q_{L}$ and $u_{i}$ are, respectively, cylinder flow and input control voltage, $K_{i}, K_{s}, K_{q}$, and $K_{c}$ are different kinds of gain listed as gain of the amplifier, servovalve coefficient, servovalve flow gain, and servovalve flow-pressure coefficient, respectively, and $p$ represents cylinder pressure difference.

2.2. Continuity Equation in the Motor. Applying the continuity equation to each chamber of motor,

$$
\begin{aligned}
& \dot{p}_{1}=\frac{\beta_{e}}{V_{0}}\left(Q_{1}-D_{m} \dot{\theta}-C_{\mathrm{ic}}\left(p_{1}-p_{2}\right)-C_{\mathrm{ec}} p_{1}\right), \\
& \dot{p}_{2}=\frac{\beta_{e}}{V_{0}}\left(-Q_{2}+D_{m} \dot{\theta}+C_{\mathrm{ic}}\left(p_{1}-p_{2}\right)-C_{\mathrm{ec}} p_{2}\right),
\end{aligned}
$$

where $D_{m}$ represents the displacement volume of motor, $\theta$ is the angle displacement of the valve plate, and $\beta_{e}, C_{\mathrm{ic}}, C_{\mathrm{ec}}$, and $V_{0}$ are coefficients defined as effective bulk modulus of system, internal or cross-port leakage coefficient of motor, external leakage coefficient of motor, and total volume of fluid 
under compression in both chambers, respectively. We define that $p_{L}=p_{1}-p_{2}$ and $Q_{L}=Q_{1}=Q_{2}$; thus,

$$
\dot{p}_{L}=Q_{L}-D_{m} \omega-Q_{l}\left(p_{1}, p_{2}, \omega\right),
$$

where $C_{\mathrm{tc}}$ means total leakage coefficient of variables in cylinder:

$$
Q_{L}=C_{d} l_{v} \sqrt{\frac{1}{2}\left(p_{s}-p_{L}\right)},
$$

where $C_{d}$ is the flow coefficient and $l_{v}$ represents the displacement of the valve.

2.3. Torque Motion Equation at the Load. The torque balance equation for the valve plate is expressed as

$$
J_{v p} \frac{d^{2} \theta}{d t^{2}}=D_{m} p_{L}-d_{r} \cdot \frac{d \theta}{d t}-T_{f}-T_{e},
$$

where $\theta$ denotes angular position of the secondary component, $J_{v p}$ represents equivalent moment of inertia of the port plate, besides, $T_{f}$ is the total friction torque, $T_{e}$ is the disturbance torque, and $d_{r}$ is the kinematic coefficient of viscosity.

\subsection{System State-Space Model. State variables are selected as} $X=\left[\begin{array}{lll}x_{1} & x_{2} & x_{3}\end{array}\right]^{T}=\left[\begin{array}{lll}\theta & \dot{\theta} & p_{L}\end{array}\right]^{T}$, where $x_{1}$ is the angular displacement of valve plate, $x_{2}$ is the rotating velocity of valve plate, and $x_{3}$ is the differential pressure of the two chambers. Thus, a state-space equation of the system under consideration can be given by

$$
\begin{gathered}
\dot{x}=\left(\begin{array}{c}
x_{2} \\
\frac{D_{m}}{J_{v p}} x_{3} \\
-\frac{D_{m} \beta_{e}}{V_{0}} x_{2}-\frac{\beta_{e} C_{e i}}{V_{0}} x_{3}
\end{array}\right)+\left[\begin{array}{c}
0 \\
0 \\
\frac{\beta_{e}}{V_{0}}
\end{array}\right] u+\left[\begin{array}{c}
0 \\
-T_{e}-T_{f} \\
0
\end{array}\right], \\
y=\left[\begin{array}{lll}
1 & 0 & 0
\end{array}\right]^{T} x .
\end{gathered}
$$

About the equation, there exist parameter uncertainty and external disturbance in the system. In detail, $\beta_{e}$ can change from $0.8 \beta_{e}$ to $1.2 \beta_{e}$, and $C_{e i}$ can also change from $0.5 C_{e i}$ to $2 C_{e i}$. For the disturbance $T_{f}$ and $T_{e}$, it is noted that $T_{f}$ is the friction torque between the cylinder block and the port plate which should be calculated in the following section to estimate the range; then, $T_{e}$ is the small disturbance caused by other factors. The main external disturbance torque is the friction torque, and the following part will focus on calculating the range of the disturbance torque.

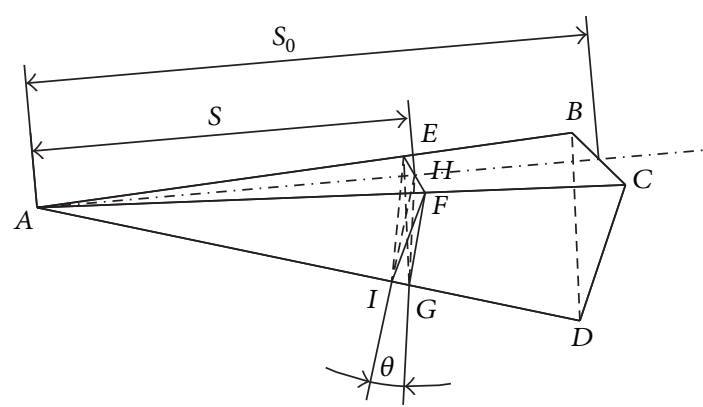

FIgURE 3: Structure of the triangular groove.

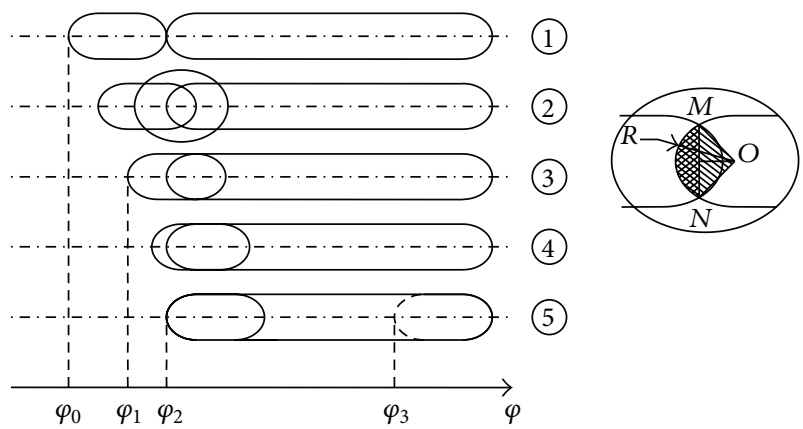

FIgURE 4: The position relationship between the plunger hole and the kidney slot.

\subsection{Range Estimation of the Disturbance Torque}

2.5.1. Calculation of Triangular Groove. The triangular groove is one common kind of dampening groove in practical engineering, which is depicted in Figure 3. It can be found that the flow area is changing with the relative location.

The flow area can be defined as the minimum area which is vertical with the flow direction; hence the flow area $A_{3}$ should be the area of $\triangle E F I$ through the geometric calculation:

$$
A_{3}=S_{\triangle E F I}=S_{\triangle E F G} \cos \theta=\frac{s}{s_{0}} \frac{\sqrt{3}}{4} a^{2} \cos \theta,
$$

where $\triangle B C D$ is the equilateral triangle and the side length is a, $\triangle A B C$ is the isosceles triangle, and in addition, the plane $A B C$ is vertical with the plane $B C D . s_{0}$ is the total length of the triangular groove and $s$ is the effective length. $\triangle E F G$ is through the $H$ point and parallel $\triangle B C D$.

2.5.2. Flow Area Calculation of the Waist Type Groove. The profile and dimensions of the three waist type grooves are all the same except for the different location. Defining the radius of the distribution circle of the waist type groove and the plunger hole is $r$. All of the three flow area calculation methods are similar. The position relationship between the hole of the plunger hole and the waist type groove during the piston is rotating as shown in Figure 4. (1), (3), and (5) are the critical positions in Figure 4 and the corresponding angles are $\varphi_{0}, \varphi_{1}$, and $\varphi_{2}$, respectively. 
(a) When $\varphi_{0}<\varphi<\varphi_{1}$, the flow area is overlying through two arches. And the area of the single arch can be obtained by subtracting the area of $\triangle O M N$ from the area of OMN.

The flow area can be obtained from

$$
\begin{aligned}
A=2\left(A^{\prime}-A^{\prime \prime}\right)= & R^{2} \arccos \left(\frac{\left(\varphi-\varphi_{0}\right) r}{2 R}\right) \\
& -\left(\varphi-\varphi_{0}\right) r \sqrt{R^{2}-\left(\frac{\left(\varphi-\varphi_{0}\right) r}{2}\right)^{2}},
\end{aligned}
$$

where the central angle is

$$
\angle M O N=2 \arccos \left(\frac{\left(\varphi-\varphi_{0}\right) r}{2 R}\right) .
$$

And the sector can be calculated as

$$
A^{\prime}=\frac{R^{2}}{2} \arccos \left(\frac{\left(\varphi-\varphi_{0}\right) r}{2 R}\right) .
$$

The area of the triangle is

$$
A^{\prime \prime}=\frac{\left(\varphi-\varphi_{0}\right) r}{2} \sqrt{R^{2}-\left(\frac{\left(\varphi-\varphi_{0}\right) r}{2}\right)^{2}} .
$$

(b) When $\varphi_{1}<\varphi<\varphi_{2}$, the flow area is equal to the difference of the entire circle area and the two sector areas; hence the flow area is

$$
A=\pi R^{2}+\frac{\varphi-\varphi_{1}}{2}\left(\left(r+\frac{R}{2}\right)^{2}-\left(r-\frac{R}{2}\right)^{2}\right) .
$$

(c) When $\varphi_{2}<\varphi<\varphi_{3}$, the entire plunger hole is contained in the waist type groove; hence the flow area is the area of the plunger hole:

$$
A=\pi R^{2}+\frac{\varphi_{\text {con }}}{2}\left(\left(r+\frac{R}{2}\right)^{2}-\left(r-\frac{R}{2}\right)^{2}\right),
$$

where $\varphi_{\text {con }}$ is the central angle of the sector of the plunger hole.

(d) When $\varphi>\varphi_{3}$ the method is the same as above; hence the period is omitted.

2.5.3. Flow Continuity Equation. Figure 5 shows the motion analysis of the axial piston pump which has piston shoes.

There are nine plungers which are doing the alternative liner motion. The radius of the distribution circle is $r$, and the plane $O x y$ is vertical with the axis of the cylinder. The plane $O^{\prime} x^{\prime} y^{\prime}$ is the same plane with the swash plate and the angle between the two planes is $\varphi$, which is the tilt angle of the swash plate. During the working period, the plungers not only rotate around the $z$-axis, but also do the liner alternating motion with the $z$-axis. Points $A$ and $B$ are the top and bottom dead points, respectively, during the plungers moving, and point $C$ is the position while the plungers at the $t$ time. Hence, the position of the plunger is

$$
\begin{gathered}
x=r \sin \alpha, \quad y=r \cos \alpha, \\
z=-r \tan \varphi \cos \alpha .
\end{gathered}
$$

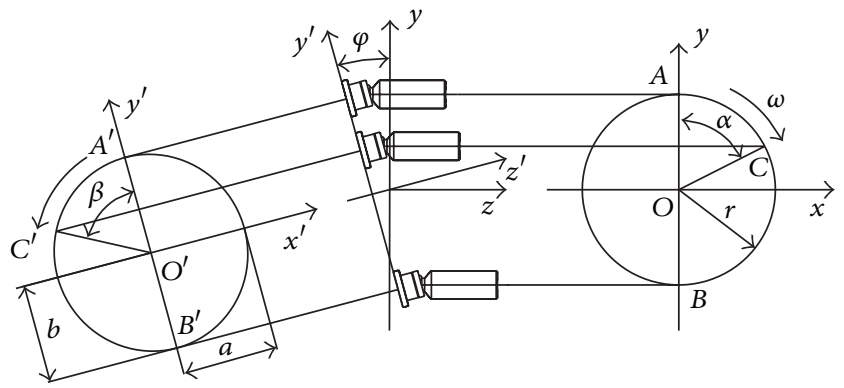

FIGURE 5: The motion analysis of the plunger.

We can also get the velocity of the plunger relative to the cylinder as follows:

$$
v=\frac{d z}{d t}=\frac{d(-r \tan \varphi \cos \alpha)}{d t}=\omega r \tan \varphi \sin \alpha,
$$

where $\omega$ is the angular velocity of the cylinder $(\mathrm{rad} / \mathrm{s})$.

Then, the flow rate of the plunger hole caused by the motion of the plunger is calculated by

$$
Q_{v}=v A_{z}=\frac{\omega \pi r d^{2}}{4} \tan \varphi \sin \alpha
$$

where $A_{z}$ is the area of the plunger $\left(\mathrm{m}^{2}\right)$ and $d$ is the diameter of the plunger $(\mathrm{m})$.

The compression flow rate inside the plunger hole can be obtained by

$$
Q_{E}=\frac{d \Delta V}{d t}=\frac{V_{0}}{E} \frac{d P}{d t}
$$

where $V_{0}$ is the oil volume corresponding to the time of $t\left(\mathrm{~m}^{3}\right)$ and $E$ is the volumetric modulus of elasticity $(\mathrm{MPa})$.

The equation for calculating the flow rate through the throttle valve is below:

$$
Q_{T}=C_{d} A \sqrt{\frac{2 \Delta P}{\rho}}
$$

where $A$ is the flow area and $\rho$ is the density of the oil and then $\Delta P$ is the pressure difference.

Then, the flow continuity equations are

$$
\begin{gathered}
Q_{v}=Q_{E}+Q_{T}, \\
\frac{\omega \pi r d^{2}}{4} \tan \varphi \sin \alpha=\frac{V_{0}}{E} \frac{d P}{d t}+C_{d} A \sqrt{\frac{2\left(p-p_{A}\right)}{\rho}}, \\
\frac{\omega \pi r d^{2}}{4} \tan \varphi \sin \alpha=\frac{V_{0}}{E} \frac{d P}{d t}+C_{d} A \sqrt{\frac{2\left(p-p_{B}\right)}{\rho}}, \\
\frac{\omega \pi r d^{2}}{4} \tan \varphi \sin \alpha=\frac{V_{0}}{E} \frac{d P}{d t}+C_{d} A \sqrt{\frac{2\left(p-p_{T}\right)}{\rho}} .
\end{gathered}
$$

The pressure inside the plunger can be got by solving the equations above. Then, the friction torque can be obtained 
by introducing the friction coefficient between the two kinds of materials. It should be noticed that the value of the friction torque maybe less than the value above because the oil film could be generated. However, it is reasonable to use this big value to design the robust controller because on one side the exact value with the influence of the oil film is difficult to calculate and the other side is that if the controller can be adaptive to the worst working condition, the controller should also work for other situations.

\section{The Main Results}

Consider the state-space model of the system with parameter uncertainties as follows:

$$
\begin{aligned}
\dot{\mathbf{x}}(t)=(\mathbf{A}+\Delta \mathbf{A}) \mathbf{x}(t)+(\mathbf{B}+\Delta \mathbf{B}) \mathbf{u}(t)+\mathbf{w}(t), \\
\mathbf{z}_{0}(t)=\mathbf{C}_{0} \mathbf{x}(t)+\mathbf{D}_{0} \mathbf{u}(t), \\
\mathbf{z}_{1}(t)=\mathbf{C}_{1} \mathbf{x}(t)+\mathbf{D}_{1} \mathbf{u}(t),
\end{aligned}
$$

where $\mathbf{x}(t)$ is the state vector, $\mathbf{u}(t)$ is the control input, $\mathbf{w}(t)$ is the disturbance input, $\mathbf{z}_{0}(t), \mathbf{z}_{1}(t)$ are the controlled outputs, $\mathbf{A}, \mathbf{B}, \mathbf{C}_{0}, \mathbf{D}_{0}, \mathbf{C}_{1}, \mathbf{D}_{1}$ are known constant matrices of appropriate dimensions, and $\Delta \mathbf{A}, \Delta \mathbf{B}$ are matrices of appropriate dimensions representing parameter uncertainties, which are supposed to be in the following form:

$$
\left[\begin{array}{ll}
\Delta \mathbf{A} & \Delta \mathbf{B}
\end{array}\right]=\mathbf{D F}(t)\left[\begin{array}{ll}
\mathbf{E}_{1} & \mathbf{E}_{2}
\end{array}\right]
$$

where $\mathbf{D}, \mathbf{E}_{1}, \mathbf{E}_{2}$ are known real constant matrices and $\mathbf{F}(t)$ is an unknown real time-varying matrix with Lebesgue measurable elements satisfying

$$
\mathbf{F}(t) \in \boldsymbol{\Omega}_{F}:=\{\mathbf{F}(t):\|\mathbf{F}(t)\| \leq \mathbf{I}\} .
$$

Assume that the desired trajectory is $\mathbf{y}_{d}(t)$ and the error vector is defined as

$$
\mathbf{e}(t)=\mathbf{y}(t)-\mathbf{y}_{d}(t)=\mathbf{x}(t)-\mathbf{y}_{d}(t)
$$

and we can obtain $\mathbf{x}(t)=\mathbf{e}(t)+\mathbf{y}_{d}(t)$. Generalized error feedback control is used to be a control input:

$$
\mathbf{u}(t)=\mathbf{K e}(t)+v(t),
$$

where $v(t)=\mathbf{B}^{+}\left(-\mathbf{A} \mathbf{y}_{d}(t)+\dot{\mathbf{y}}_{d}(t)\right)$ and $\mathbf{B}^{+}$is the pseudoinverse of matrix B. According to above equations, the following equation can be obtained:

$$
\dot{\mathbf{e}}(t)=(\overline{\mathbf{A}}+\overline{\mathbf{B}} \mathbf{K}) \mathbf{e}(t)+\mathbf{w}(t) .
$$

Define $\mathbf{u}_{e}=\mathbf{u}(t)-v(t)$; the cost function associated with the system (21) is

$$
\mathbf{J}_{e}=\int_{0}^{\infty} \mathbf{e}^{T}(t) \mathbf{Q} \mathbf{e}(t)+\mathbf{u}_{e}^{T}(t) \mathbf{R} \mathbf{u}_{e}(t) d t
$$

where $\mathbf{Q}$ and $\mathbf{R}$ are the weighing positive matrices.
The purpose of this paper is to study an $\mathbf{H}_{2} / \mathbf{H}_{\infty}$ guaranteed cost control for all admissible uncertainties such that

(1) the closed-loop system is asymptotically stable;

(2) under zero initial condition, the closed-loop system guarantees that $\|\mathbf{z}\|_{2}<\gamma\|\mathbf{w}\|_{2}$ for all nonzero $\mathbf{w} \in$ $\mathbf{L}_{2}[0, \infty)$ and a prescribed scalar $\gamma>0$;

(3) the input constraint (7) is satisfied.

For obtaining the main conclusions of this paper, the following lemma will be adopted to handle the parameter uncertainties in the system.

Lemma 1. Given matrices with appropriate dimensions $\mathbf{Y}, \mathbf{C}$, and $\mathbf{D}$ and that $\mathbf{Y}$ is the symmetric matrix, then

$$
\mathbf{Y}+\mathbf{C F D}+\mathbf{D}^{T} \mathbf{F}^{T} \mathbf{C}^{T}<0
$$

For all $\mathbf{F}$ satisfying $\mathbf{F}^{T} \mathbf{F} \leq \mathbf{I}$, if and only if there exists a scalar $\varepsilon>0$ such that

$$
\mathbf{Y}+\varepsilon \mathbf{C C}^{T}+\varepsilon^{-1} \mathbf{D}^{T} \mathbf{D}<0 .
$$

Theorem 2. Given constant $\gamma>0$, if there exist scalars $\alpha>0$, $\beta>0, \mathbf{P}=\mathbf{P}^{T}>0$ satisfying the following inequality:

$$
\begin{aligned}
(\mathbf{A}+ & \mathbf{B K})^{T} \mathbf{P}+\mathbf{P}(\mathbf{A}+\mathbf{B K})+\mathbf{P}\left(\boldsymbol{\alpha} \mathbf{D D}^{T}+\boldsymbol{\beta} \boldsymbol{\gamma}^{-2} \mathbf{B}_{\mathbf{w}} \mathbf{B}_{\mathbf{w}}^{T}\right) \mathbf{P} \\
& +\boldsymbol{\alpha}^{-1}\left(\mathbf{E}_{1}+\mathbf{E}_{2} \mathbf{K}\right)^{T}\left(\mathbf{E}_{1}+\mathbf{E}_{2} \mathbf{K}\right) \\
& +\boldsymbol{\beta}^{-1}\left(\mathbf{C}_{1}+\mathbf{D}_{1} \mathbf{K}\right)^{T}\left(\mathbf{C}_{1}+\mathbf{D}_{1} \mathbf{K}\right) \\
& +\left(\mathbf{C}_{0}+\mathbf{D}_{0} \mathbf{K}\right)^{T}\left(\mathbf{C}_{0}+\mathbf{D}_{0} \mathbf{K}\right)<0,
\end{aligned}
$$

then the controller in the form of (25) exists, such that

(1) the closed-loop system (21) with $\mathbf{w}(t)=0$ is asymptotically stable;

(2) under zero initial condition, the closed-loop system guarantees that $\|\mathbf{z}\|_{2}<\gamma\|\mathbf{w}\|_{2}$ for all nonzero $\mathbf{w} \in$ $\mathbf{L}_{2}[0, \infty)$ and a prescribed scalar $\gamma>0$;

(3) if (30) has a symmetric positive definite solution $\mathbf{P}$, then for all admissible parameter uncertainties, $0 \leq \overline{\mathbf{P}} \leq \mathbf{P}$, where $\overline{\mathbf{P}}=\overline{\mathbf{P}}^{T} \geq 0$ is a solution to Lyapunov equation:

$$
\begin{aligned}
\mathbf{P}\left(\mathbf{A}+\mathbf{D F}(t)\left(\mathbf{E}_{1}+\mathbf{E}_{2} \mathbf{K}\right)\right)+\left(\mathbf{A}+\mathbf{D F}(t)\left(\mathbf{E}_{1}+\mathbf{E}_{2} \mathbf{K}\right)\right)^{T} \\
+\left(\mathbf{C}_{0}+\mathbf{D}_{0} \mathbf{K}\right)^{T}\left(\mathbf{C}_{0}+\mathbf{D}_{0} \mathbf{K}\right)=0 .
\end{aligned}
$$

Proof. From [23], it follows that system (21) with $\mathbf{w}(t)=0$ is asymptotically stable and $\|\mathbf{z}\|_{2}<\gamma\|\mathbf{w}\|_{2}$ if there exists a symmetric positive definite matrix $\overline{\mathbf{P}}$ such that

$$
\begin{aligned}
\left(\mathbf{A}+\mathbf{B K}+\mathbf{D F}(t)\left(\mathbf{E}_{1}+\mathbf{E}_{2} \mathbf{K}\right)\right)^{T} \overline{\mathbf{P}} \\
\quad+\overline{\mathbf{P}}\left(\mathbf{A}+\mathbf{B K}+\mathbf{D F}(t)\left(\mathbf{E}_{1}+\mathbf{E}_{2} \mathbf{K}\right)\right)+\gamma^{-2} \overline{\mathbf{P}} \mathbf{B}_{\mathbf{w}} \mathbf{B}_{\mathbf{w}}^{T} \overline{\mathbf{P}} \\
\quad+\left(\mathbf{C}_{1}+\mathbf{D}_{1} \mathbf{K}\right)^{T}\left(\mathbf{C}_{1}+\mathbf{D}_{1} \mathbf{K}\right)<0 .
\end{aligned}
$$


The above inequality is equivalent to the fact that there exists a scalar $\beta>0$ such that

$$
\begin{aligned}
\left(\mathbf{A}+\mathbf{B K}+\mathbf{D F}(t)\left(\mathbf{E}_{1}+\mathbf{E}_{2} \mathbf{K}\right)\right)^{T} \overline{\mathbf{P}} \\
\quad+\overline{\mathbf{P}}\left(\mathbf{A}+\mathbf{B K}+\mathbf{D F}(t)\left(\mathbf{E}_{1}+\mathbf{E}_{2} \mathbf{K}\right)\right) \\
\quad+\boldsymbol{\gamma}^{-2} \overline{\mathbf{P}} \mathbf{B}_{\mathbf{w}} \mathbf{B}_{\mathbf{w}}^{T} \overline{\mathbf{P}}+\left(\mathbf{C}_{1}+\mathbf{D}_{1} \mathbf{K}\right)^{T}\left(\mathbf{C}_{1}+\mathbf{D}_{1} \mathbf{K}\right) \\
\quad+\boldsymbol{\beta}\left(\mathbf{C}_{0}+\mathbf{D}_{0} \mathbf{K}\right)^{T}\left(\mathbf{C}_{0}+\mathbf{D}_{0} \mathbf{K}\right)<0 .
\end{aligned}
$$

Define $\mathbf{P}=\beta^{-1} \overline{\mathbf{P}}$; the above inequality can be described as

$$
\begin{aligned}
(\mathbf{A}+ & \mathbf{B K})^{T} \mathbf{P}+\mathbf{P}(\mathbf{A}+\mathbf{B K}) \\
& +\boldsymbol{\beta} \gamma^{-2} \mathbf{P} \mathbf{B}_{\mathbf{w}} \mathbf{B}_{\mathbf{w}}^{T} \mathbf{P}+\boldsymbol{\beta}^{-1}\left(\mathbf{C}_{1}+\mathbf{D}_{1} \mathbf{K}\right)^{T}\left(\mathbf{C}_{1}+\mathbf{D}_{1} \mathbf{K}\right) \\
& +\left(\mathbf{C}_{0}+\mathbf{D}_{0} \mathbf{K}\right)^{T}\left(\mathbf{C}_{0}+\mathbf{D}_{0} \mathbf{K}\right)+\mathbf{P D F}\left(\mathbf{E}_{1}+\mathbf{E}_{2} \mathbf{K}\right) \\
& +\left(\mathbf{E}_{1}+\mathbf{E}_{2} \mathbf{K}\right)^{T} \mathbf{F}^{T} \mathbf{D}^{T} \mathbf{P}<0 .
\end{aligned}
$$

According to Lemma 1, the above inequality holds for all F satisfying $\mathbf{F}^{T} \mathbf{F} \leq \mathbf{I}$ if and only if there exists $\alpha>0$ such that

$$
\begin{aligned}
(\mathbf{A}+\mathbf{B K})^{T} \mathbf{P}+\mathbf{P}(\mathbf{A}+\mathbf{B K})+\boldsymbol{\beta} \boldsymbol{\gamma}^{-2} \mathbf{P} \mathbf{B}_{\mathbf{w}} \mathbf{B}_{\mathbf{w}}^{T} \mathbf{P} \\
\quad+\boldsymbol{\beta}^{-1}\left(\mathbf{C}_{1}+\mathbf{D}_{1} \mathbf{K}\right)^{T}\left(\mathbf{C}_{1}+\mathbf{D}_{1} \mathbf{K}\right) \\
\quad+\left(\mathbf{C}_{0}+\mathbf{D}_{0} \mathbf{K}\right)^{T}\left(\mathbf{C}_{0}+\mathbf{D}_{0} \mathbf{K}\right) \\
\quad+\boldsymbol{\alpha} \mathbf{P D D}^{T} \mathbf{P}+\boldsymbol{\alpha}^{-1}\left(\mathbf{E}_{1}+\mathbf{E}_{2} \mathbf{K}\right)^{T}\left(\mathbf{E}_{1}+\mathbf{E}_{2} \mathbf{K}\right)<0
\end{aligned}
$$

So, we obtain (30). Suppose that (30) has a symmetric positive definite solution $\mathbf{P}$, define

$$
\begin{aligned}
\boldsymbol{\Delta}= & \boldsymbol{\alpha} \mathbf{P D D}^{T} \mathbf{P}+\boldsymbol{\alpha}^{-1}\left(\mathbf{E}_{1}+\mathbf{E}_{2} \mathbf{K}\right)^{T}\left(\mathbf{E}_{1}+\mathbf{E}_{2} \mathbf{K}\right) \\
& -\operatorname{PDF}\left(\mathbf{E}_{1}+\mathbf{E}_{2} \mathbf{K}\right)-\left(\mathbf{E}_{1}+\mathbf{E}_{2} \mathbf{K}\right)^{T} \mathbf{F}^{T} \mathbf{D}^{T} \mathbf{P} .
\end{aligned}
$$

According to [24] and considering $\|\mathbf{F}(t)\| \leq \mathbf{I}$, it follows that $\Delta \geq 0$; then subtracting

$$
\begin{aligned}
\mathbf{P}\left(\mathbf{A}+\mathbf{D F}(t)\left(\mathbf{E}_{1}+\mathbf{E}_{2} \mathbf{K}\right)\right)+\left(\mathbf{A}+\mathbf{D F}(t)\left(\mathbf{E}_{1}+\mathbf{E}_{2} \mathbf{K}\right)\right)^{T} \\
+\left(\mathbf{C}_{0}+\mathbf{D}_{0} \mathbf{K}\right)^{T}\left(\mathbf{C}_{0}+\mathbf{D}_{0} \mathbf{K}\right)=0
\end{aligned}
$$

from (30), we can get

$$
\begin{aligned}
\left(\mathbf{A}+\mathbf{B K}+\mathbf{D F}(t)\left(\mathbf{E}_{1}+\mathbf{E}_{2} \mathbf{K}\right)\right)^{T}(\mathbf{P}-\overline{\mathbf{P}}) \\
\quad+(\mathbf{P}-\overline{\mathbf{P}})\left(\mathbf{A}+\mathbf{B K}+\mathbf{D F}(t)\left(\mathbf{E}_{1}+\mathbf{E}_{2} \mathbf{K}\right)\right) \\
\quad+\boldsymbol{\beta} \boldsymbol{\gamma}^{-2} \mathbf{P B}_{\mathbf{w}} \mathbf{B}_{\mathbf{w}}^{T} \mathbf{P}+\boldsymbol{\beta}^{-1}\left(\mathbf{C}_{1}+\mathbf{D}_{1} \mathbf{K}\right)^{T}\left(\mathbf{C}_{1}+\mathbf{D}_{1} \mathbf{K}\right) \\
\quad+\boldsymbol{\Delta}<0 .
\end{aligned}
$$

Since $\boldsymbol{\beta} \boldsymbol{\gamma}^{-2} \mathbf{P} \mathbf{B}_{\mathbf{w}} \mathbf{B}_{\mathbf{w}}^{T} \mathbf{P}+\boldsymbol{\beta}^{-1}\left(\mathbf{C}_{1}+\mathbf{D}_{1} \mathbf{K}\right)^{T}\left(\mathbf{C}_{1}+\mathbf{D}_{1} \mathbf{K}\right)+\boldsymbol{\Delta} \geq 0$ and system (21) with $\mathbf{w}(t)=0$ is asymptotically stable according to the Lyapunov stability theory, we can get $\mathbf{P}-\overline{\mathbf{P}} \geq 0$ or $\overline{\mathbf{P}} \leq \mathbf{P}$; then the proof is completed.

If there exist two scalars $\alpha>0, \beta>0$ such that the matrix inequality (30) has a symmetric positive definite solution $\mathbf{P}$, then the closed-loop system is asymptotically stable for all admissible parameter uncertainties and satisfies an $\mathbf{H}_{\infty}$ disturbance attenuation constraint. In addition, this solution $\mathbf{P}$ will guarantee the worst $\mathbf{H}_{2}$ performance index that satisfies $\mathbf{J}_{0}(\mathbf{K}) \leq \mathbf{J}(\mathbf{K}, \mathbf{P})=\operatorname{tr}\left(\mathbf{B}_{\mathbf{w}}^{T} \mathbf{P B}_{\mathbf{w}}\right) \cdot \mathbf{J}(\mathbf{K}, \mathbf{P})$ is considered as an $\mathbf{H}_{2} / \mathbf{H}_{\infty}$ guaranteed cost bound of the closed-loop system.

Theorem 3. Given a constant $\gamma>0$, the system (21) is asymptotically stable and satisfies $\|\mathbf{z}\|_{2}<\gamma\|\mathbf{w}\|_{2}$ for any nonzero $\mathbf{w} \in \mathbf{L}_{2}[0, \infty)$, and the input constraint is guaranteed if there exist scalars $\alpha>0, \beta>0$, a symmetric positive definite matrix $\mathbf{X}$, and a matrix $\mathbf{W}$ satisfying

$$
\begin{gathered}
{\left[\begin{array}{cccc}
\mathbf{V} & \left(\mathbf{E}_{1} \mathbf{X}+\mathbf{E}_{2} \mathbf{W}\right)^{T} & \left(\mathbf{C}_{1} \mathbf{X}+\mathbf{D}_{1} \mathbf{W}\right)^{T} & \left(\mathbf{C}_{0} \mathbf{X}+\mathbf{D}_{0} \mathbf{W}\right)^{T} \\
\mathbf{E}_{1} \mathbf{X}+\mathbf{E}_{2} \mathbf{W} & -\boldsymbol{\alpha} \mathbf{I} & 0 & 0 \\
\mathbf{C}_{1} \mathbf{X}+\mathbf{D}_{1} \mathbf{W} & 0 & -\beta \mathbf{I} & 0 \\
\mathbf{C}_{0} \mathbf{X}+\mathbf{D}_{0} \mathbf{W} & 0 & 0 & -\mathbf{I}
\end{array}\right]<0} \\
{\left[\begin{array}{cc}
-\mathbf{I} & \sqrt{\rho} \mathbf{W} \\
* & -\mathbf{u}_{\max }^{2} \mathbf{P}
\end{array}\right]<0,}
\end{gathered}
$$

where $\mathbf{V}=(\mathbf{A X}+\mathbf{B W})^{T}+\mathbf{A X}+\mathbf{B W}+\boldsymbol{\alpha} \mathbf{D D}^{T}+\boldsymbol{\beta} \boldsymbol{\gamma}^{-2} \mathbf{B}_{\mathbf{w}} \mathbf{B}_{\mathbf{w}}^{T}$. In addition, if the above inequalities have a feasible solution $(\boldsymbol{\alpha}, \boldsymbol{\beta}, \mathbf{X}, \mathbf{W})$, the state feedback controller can be given by $\mathbf{u}(t)=$ $\mathbf{W X}^{-1} \mathbf{e}(t)+v(t)$, and the $\mathbf{H}_{2} / \mathbf{H}_{\infty}$ guaranteed cost bound of the closed-loop system is described by $\mathbf{J}\left(\mathbf{K}, \mathbf{X}^{-1}\right)=\operatorname{tr}\left(\mathbf{B}_{\mathbf{w}}^{T} \mathbf{X}^{-1} \mathbf{B}_{\mathbf{w}}\right)$.
Proof. It follows from Theorem 2 that there exists a controller $\mathbf{u}(t)=\mathbf{K e}(t)+v(t)$ such that the design criteria (1) and (2) are satisfied if and only if there exist two scalars $\alpha>0, \beta>0$ and a symmetric positive definite matrix $\mathbf{P}$ which guarantees that the matrix inequality (30) holds. Pre- and postmultiplying 


$$
\left[\begin{array}{cccc}
\overline{\mathbf{V}} & \mathbf{P}^{-1}\left(\mathbf{E}_{1} \mathbf{X}+\mathbf{E}_{2} \mathbf{W}\right)^{T} & \mathbf{P}^{-1}\left(\mathbf{C}_{1} \mathbf{X}+\mathbf{D}_{1} \mathbf{W}\right)^{T} & \mathbf{P}^{-1}\left(\mathbf{C}_{0} \mathbf{X}+\mathbf{D}_{0} \mathbf{W}\right)^{T} \\
\left(\mathbf{E}_{1} \mathbf{X}+\mathbf{E}_{2} \mathbf{W}\right) \mathbf{P}^{-1} & -\boldsymbol{\alpha} \mathbf{I} & 0 & 0 \\
\left(\mathbf{C}_{1} \mathbf{X}+\mathbf{D}_{1} \mathbf{W}\right) \mathbf{P}^{-1} & 0 & -\beta \mathbf{I} & 0 \\
\left(\mathbf{C}_{0} \mathbf{X}+\mathbf{D}_{0} \mathbf{W}\right) \mathbf{P}^{-1} & 0 & 0 & -\mathbf{I}
\end{array}\right]<0
$$

where $\overline{\mathbf{V}}=\mathbf{P}^{-1}(\mathbf{A}+\mathbf{B K})^{T}+(\mathbf{A}+\mathbf{B K}) \mathbf{P}^{-1}+\boldsymbol{\alpha} \mathbf{D D}^{T}+\boldsymbol{\beta} \gamma^{-2} \mathbf{B}_{\mathbf{w}} \mathbf{B}_{\mathbf{w}}^{T}$. Defining $\mathbf{X}=\mathbf{P}^{-1}, \mathbf{W}=\mathbf{K P}^{-1}$, the matrix inequality (39) can be easily obtained from (42).

In addition, we will show that the input constraint is satisfied. Define $\mathbf{V}=\mathbf{e}^{T} \mathbf{P e}$; we can easily know $\dot{\mathbf{V}}<0$ and thus $\mathbf{e}^{T} \mathbf{P e} \leq \mathbf{e}_{0}^{T} \mathbf{P e}$; we can also obtain

$$
\begin{aligned}
\max _{t>0}|\mathbf{u}(t)|^{2} & <\max _{t>d(t)}\left\|\mathbf{e}^{T}(t) \mathbf{K}^{T} \mathbf{K e}(t)\right\|_{2} \\
& =\max _{t>d(t)}\left\|\mathbf{e}^{T}(t) \mathbf{P}^{1 / 2} \mathbf{P}^{-1 / 2} \mathbf{K}^{T} \mathbf{K} \mathbf{P}^{-1 / 2} \mathbf{P}^{1 / 2} \mathbf{e}(t)\right\|_{2} \\
& <\rho \cdot \theta_{\max }\left(\mathbf{P}^{-1 / 2} \mathbf{K}^{T} \mathbf{K} \mathbf{P}^{-1 / 2}\right),
\end{aligned}
$$

where $\theta_{\max }(\cdot)$ shows the maximal eigenvalue. From the above inequality, the input constraint is established if $\rho \mathbf{P}^{-1 / 2} \mathbf{K}^{T} \mathbf{K} \mathbf{P}^{-1 / 2}<\mathbf{u}_{\max }^{2}$ I. By Schur complements, the above inequality is equivalent to (41). This completes the proof.

Theorem 3 provides a characterization of all controllers that guarantee the design criteria (1) and (2) to be achieved, and the controller $\mathbf{u}(t)=\mathbf{W} \mathbf{X}^{-1} \mathbf{e}(t)+v(t)$ provides a $\mathbf{H}_{2} / \mathbf{H}_{\infty}$ guaranteed cost bound $\operatorname{tr}\left(\mathbf{B}_{\mathbf{w}}^{T} \mathbf{X}^{-1} \mathbf{B}_{\mathbf{w}}\right)$.

\section{Simulation Results}

According to (7) combined with the uncertain parameter, consider the system with the following state-space matrices:

$$
\begin{aligned}
& \dot{x}(t)=\left(\begin{array}{ccc}
0 & 1 & 0 \\
0 & 0 & 0.0085 \\
0 & -1.68 e 8 & -1.7
\end{array}\right) x(t) \\
&+\left(\begin{array}{ccc}
0 & 0 & 0 \\
0 & 0 & 0 \\
0 & -0.85 e 8 & -0.856
\end{array}\right) x(t) \\
&+\left[\begin{array}{c}
0 \\
0 \\
3.41 e 12
\end{array}\right] u(t)+\left[\begin{array}{c}
0 \\
1.71 e 12
\end{array}\right] u(t)+\left[\begin{array}{c}
0 \\
-T_{e}-T_{f} \\
0
\end{array}\right] \\
& z_{0}(t)=\left[\begin{array}{lll}
1 & 1 & 1
\end{array}\right] x(t)+u(t) \\
& z_{1}(t)=\left[\begin{array}{lll}
1 & 0 & 0
\end{array}\right] x(t),
\end{aligned}
$$

both sides of (30) by $\mathbf{P}^{-1}$ and using Schur's complement yield that (30) is equivalent to

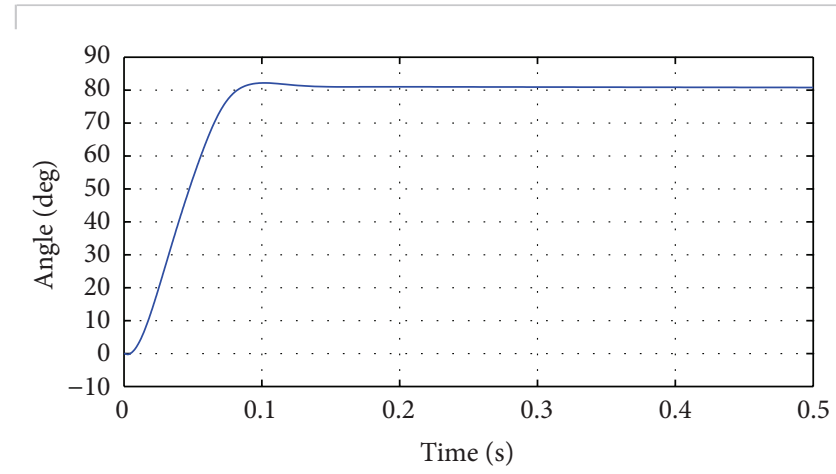

FIGURE 6: Step response of the PI control.

with

$$
\begin{gathered}
\mathbf{D}=\left[\begin{array}{lll}
0 & 0 & 0 \\
0 & 0 & 0 \\
1 & 1 & 1
\end{array}\right], \quad \mathbf{E}_{1}=\left[\begin{array}{ccc}
0 & 0 & 0 \\
0 & 0 & 0 \\
0 & -0.85 e 8 & -0.856
\end{array}\right], \\
\mathbf{E}_{2}=\left[\begin{array}{c}
0 \\
0 \\
1.71 e 12
\end{array}\right], \\
\mathbf{F}=\left[\begin{array}{lll}
r_{1} & & \\
& r_{2} & \\
& & s_{1}
\end{array}\right], \quad-1 \leq r_{1}, r_{2}, s_{1} \leq 1 .
\end{gathered}
$$

The angle displacement control system of the port plate is simulated according to the result above and the comparison with the traditional PI control is conducted. During the working condition of HT, the port plate angle should change to meet the requirement of the servosystem. Hence, the extreme working condition is the period that the port plate angle is varying because the disturbance, which is the friction torque mainly, is varying greatly. The other working conditions are chosen as the step response to reveal the dynamic response. The model is constructed in Simulink and Figures 6-9 show the simulation result.

According to Figures 6 and 7, it can be found that the step responses of both the two control methods are fast to meet the practical requirement. PI control can get a faster response; however, the overshot is larger. The GCC does not show the obvious advantage to the PI control. This is because the friction torque disturbance acts the least influence compared with the changing working condition. Hence, the good performance can be got by adjusting PI coefficients. However, the coefficients should be changed corresponding to different 


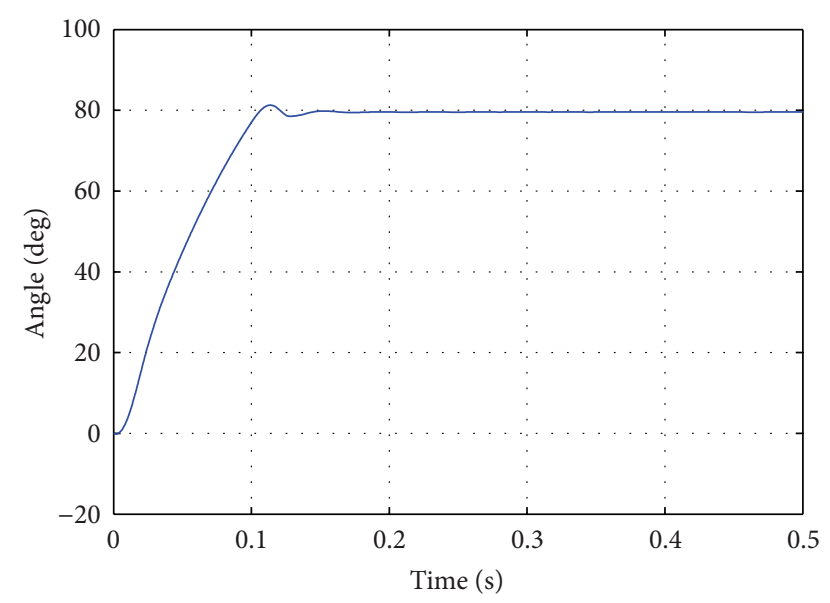

FIGURE 7: Step response of the GCC.

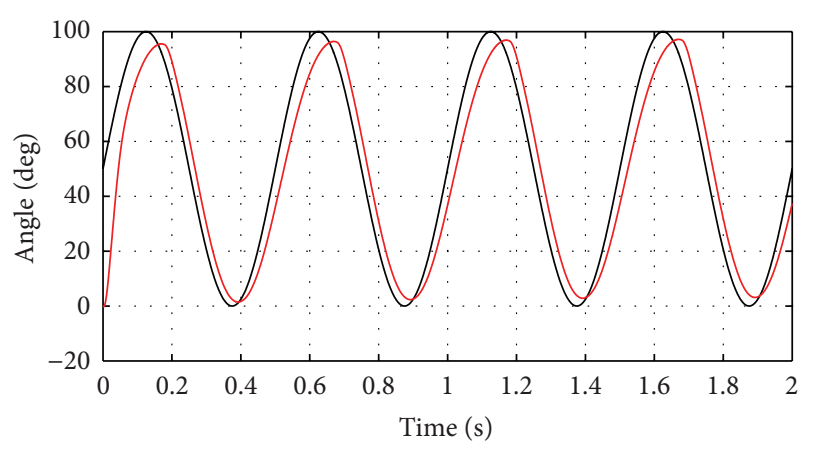

Figure 8: Sinusoidal signal response of the PI control.

loads, which is difficult in practice. This phenomenon can be explained by using sinusoidal response simulation. The sinusoidal signal responses are depicted by Figures 8 and 9 . The black curve is the command signal and the red curve is the response. The GCC follows the command much better than the traditional PI control. The results can be shown clearly through Figures 10 and 11 which show the tracking error of the two control methods. The reason is mainly about the better robustness of the GCC. The improvement over the traditional PI control is dramatic.

\section{Conclusion}

This paper is focused on the robust control for the port plate angle displacement control system with parameter uncertainties and load disturbance. The critical problem about the angle displacement control is analyzed and the main disturbance range is calculated by studying the stress relationship between the port plate and the cylinder. The analysis result shows that the friction torque is changing with different port plate angles, differential pressures, and the rotating speed. Furthermore, the guaranteed cost control for the system is designed. Simulation results show that both the GCC and PI control can get a good response under running the step response simulation. Especially, the GCC achieves much better robustness than PI control during the sinusoidal signal response simulation which is because the disturbance

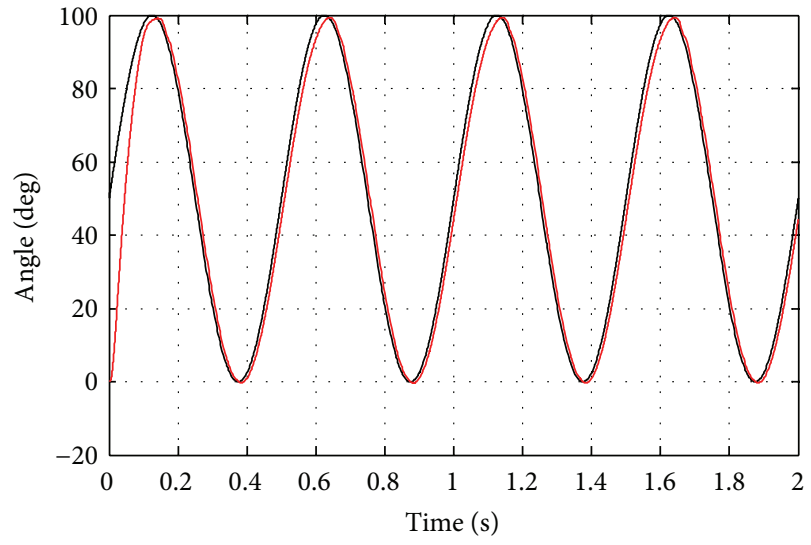

FIgURE 9: Sinusoidal signal response of the GCC.

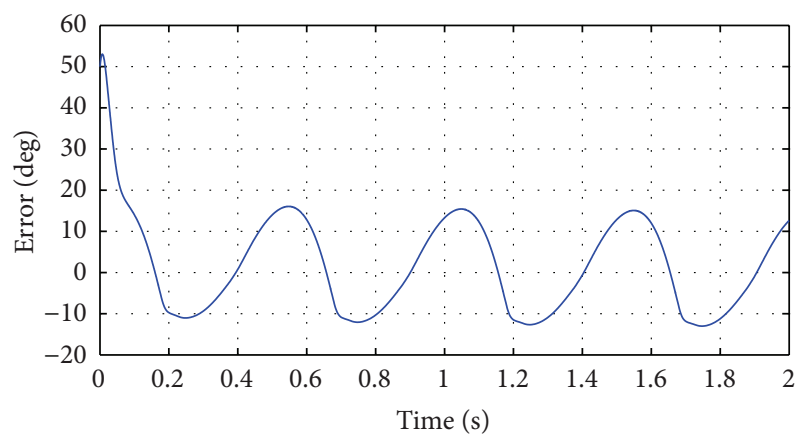

FIGURE 10: Tracking error of the PI control.

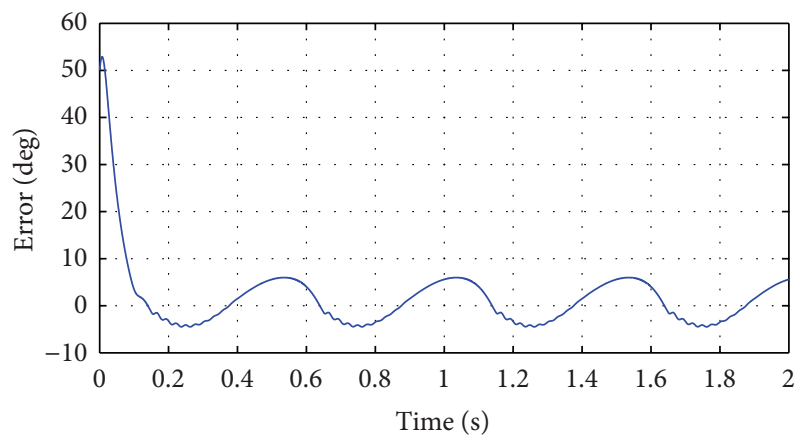

FIgURE 11: Tracking error of the GCC.

changes greatly is while the port plate is moving all the time. In order to prove the effectiveness of the proposed control method, the next step for this project is to construct the test rig.

\section{Conflict of Interests}

The authors declare that there is no conflict of interests regarding the publication of this paper.

\section{Acknowledgments}

The authors acknowledge the contribution of the National Natural Science Foundation of China (50875054, 51275123) 
and open fund of State Key Laboratory of Fluid Power Transmission and Control, Zhejiang University (GZKF-2008003).

\section{References}

[1] I. Y. Jong, K. K. Ahn, and Q. T. Dinh, "A study on an energy saving electro-hydraulic excavator," in Proceedings of the ICROS-SICE International Joint Conference (ICCAS-SICE '09), pp. 3825-3830, August 2009.

[2] J. Yang, L. Quan, and Y. Yang, "Excavator energy-saving efficiency based on diesel engine cylinder deactivation technology," Chinese Journal of Mechanical Engineering, vol. 25, no. 5, pp. 897-904, 2012.

[3] T. Lin, Q. Wang, B. Hu, and W. Gong, "Development of hybrid powered hydraulic construction machinery," Automation in Construction, vol. 19, no. 1, pp. 11-19, 2010.

[4] H. Kim, J. Choi, and K. Yi, "Development of supervisory control strategy for optimized fuel consumption of the compound hybrid excavator," Journal of Automobile Engineering, vol. 226, no. 12, pp. 1652-1666, 2012.

[5] K. K. Ahn, H. O. TH, and Q. T. Dinh, "A study on energy saving potential of hydraulic on energy saving potential of hydraulic control system using switching type closed loop constant pressure system," in Proceedings of the 7th JFPS International Symposium on Fluid Power, pp. 317-322, 2008.

[6] Q. Xiao, Q. Wang, and Y. Zhang, "Control strategies of power system in hybrid hydraulic excavator," Automation in Construction, vol. 17, no. 4, pp. 361-367, 2008.

[7] S. Sgro, M. Inderelst, and H. Murrenhoff, "Energy efficiency of mobile working machines," in Proceedings of the 7th International Fluid Power Conference, 2010.

[8] J. Jiang, A. Yu, and W. Shen, "The review of full hydraulic hybrid excavator based on common pressure rail network," Chinese Hydraulics and Pneumatics, vol. 9, pp. 44-49, 2010.

[9] P. Achten, G. Vael, H. Murrenhoff, T. Kohmäscher, and M. Inderelst, "Low-emission hydraulic hybrid for passenger cars," Automobiltechnische Zeitschrift, vol. 111, pp. 378-384, 2009.

[10] W. Shen and J. Jiang, "Analysis and development of the hydraulic secondary regulation system based on the CPR," in Proceedings of the International Conference on Fluid Power and Mechatronics (FPM '11), pp. 117-122, August 2011.

[11] P. A. J. Achten, G. E. M. Vael, and F. Zhao, "The Innas hydraulic transformer-the key to the hydrostatic common pressure rail," SAE paper 2000-01-2561.

[12] W. Shen and J.-H. Jiang, "Analysis of energy recovery efficiency of hydraulic hybrid excavator," Journal of South China University of Technology, vol. 40, no. 1, pp. 82-87, 2012.

[13] P. A. J. Achten, F. Zhao, and G. E. M. Vael, “Transforming future hydraulics: a new design of a hydraulic transformer," in Proceedings of the The 5th Scandinavian International Conference on Fluid Power, 1997.

[14] W. Shen, J. Jiang, X. Su, and H. R. Karimi, "Energy-saving analysis of hydraulic hybrid excavator based on common pressure rail," The Scientific World Journal, vol. 2013, Article ID 560694, 12 pages, 2013.

[15] H. Zhang, Y. Shi, and A. Saadat Mehr, "Robust static output feedback control and remote PID design for networked motor systems," IEEE Transactions on Industrial Electronics, vol. 58, no. 12, pp. 5396-5405, 2011.

[16] H. Li, H. Liu, H. Gao, and P. Shi, "Reliable fuzzy control for active suspension systems with actuator delay and fault," IEEE Transactions on Fuzzy Systems, vol. 20, no. 2, pp. 342-357, 2012.
[17] Z. Wang, D. W. C. Ho, Y. Liu, and X. Liu, "Robust $H_{\infty}$ control for a class of nonlinear discrete time-delay stochastic systems with missing measurements," Automatica, vol. 45, no. 3, pp. 684-691, 2009.

[18] J. Jiang, Y. Han, and D. Wang, "The intelligent PID control in Hydrostatic drive system with secondary regulation," Journal of Harbin Institute of Technology, vol. 30, pp. 36-38, 1998.

[19] H. Li, J. Yu, C. Hilton, and H. Liu, "Adaptive sliding mode control for nonlinear active suspension vehicle systems using TS fuzzy approach," IEEE Transactions on Industrial Electronics, vol. 60, no. 8, pp. 3328-3338, 2013.

[20] C. S. Kim and C. O. Lee, "Robust speed control of a variabledisplacement hydraulic motor considering saturation nonlinearity," Journal of Dynamic Systems, Measurement and Control, Transactions of the ASME, vol. 122, no. 1, pp. 196-201, 2000.

[21] W. Shen, J. Jiang, and H. R. Karimi, "Observer-based robust control for hydraulic velocity control system," Mathematical Problems in Engineering, vol. 2013, Article ID 689132, 9 pages, 2013.

[22] S. Zhou and W. X. Zheng, "Robust $H_{\infty}$ control of delayed singular systems with linear fractional parametric uncertainties," Journal of the Franklin Institute, vol. 346, no. 2, pp. 147-158, 2009.

[23] A. Kato and K. Ohnishi, "Robust force sensorless control in motion control system," in Proceedings of the 9th IEEE International Workshop on Advanced Motion Control, pp. 165170, March 2006.

[24] H. Zhang, J. Wang, and Y. Wang, "Robust filtering for ammonia coverage estimation in Diesel engine selective catalytic reduction (SCR) Systems," ASME Transactions, Journal of Dynamic Systems, Measurement, and Control, vol. 135, no. 6, Article ID 064504, 7 pages, 2013.

[25] S. Xu and J. Lam, "Guaranteed cost control," Lecture Notes in Control and Information Science, vol. 332, pp. 107-118, 2006.

[26] S. O. R. Moheimani and I. R. Petersen, "Optimal guaranteed cost control of uncertain systems via static and dynamic output feedback," Automatica, vol. 32, no. 4, pp. 575-579, 1996.

[27] J. Doyle, K. Zhou, K. Glover, and B. Bodenheimer, "Mixed $H_{2}$ and $H_{\infty}$ performance objectives II: optimal control," IEEE Transactions on Automatic Control, vol. 39, no. 8, pp. 1575-1587, 1994.

[28] J. Abedor, K. Nagpal, P. P. Khargonekar, and K. Poolla, "Robust regulation in the presence of norm-bounded uncertainty," IEEE Transactions on Automatic Control, vol. 40, no. 1, pp. 147-153, 1995.

[29] J. Ren and Q. Zhang, "Robust normalization and guaranteed cost control for a class of uncertain descriptor systems," Automatica, vol. 48, pp. 1693-1697, 2012.

[30] H. Mukaidani, "An LMI approach to guaranteed cost control for uncertain delay systems," IEEE Transactions on Circuits and Systems I, vol. 50, no. 6, pp. 795-800, 2003.

[31] P. A. J. Achten and J. O. Palmberg, "What a difference a hole makes: the commercial value of the innas hydraulic transformer," in Proceedings of the 6th Scandinavian International Conference on Fluid Power, pp. 873-886, 1999. 


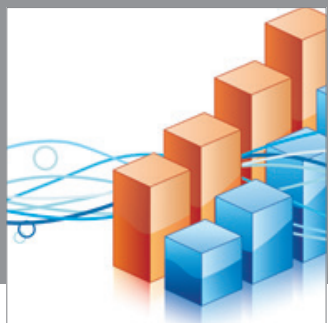

Advances in

Operations Research

mansans

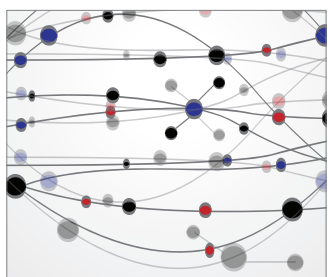

The Scientific World Journal
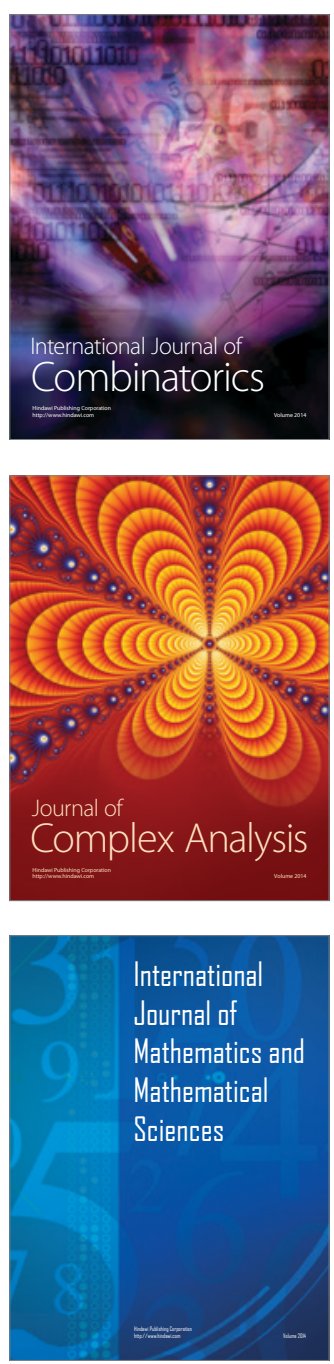
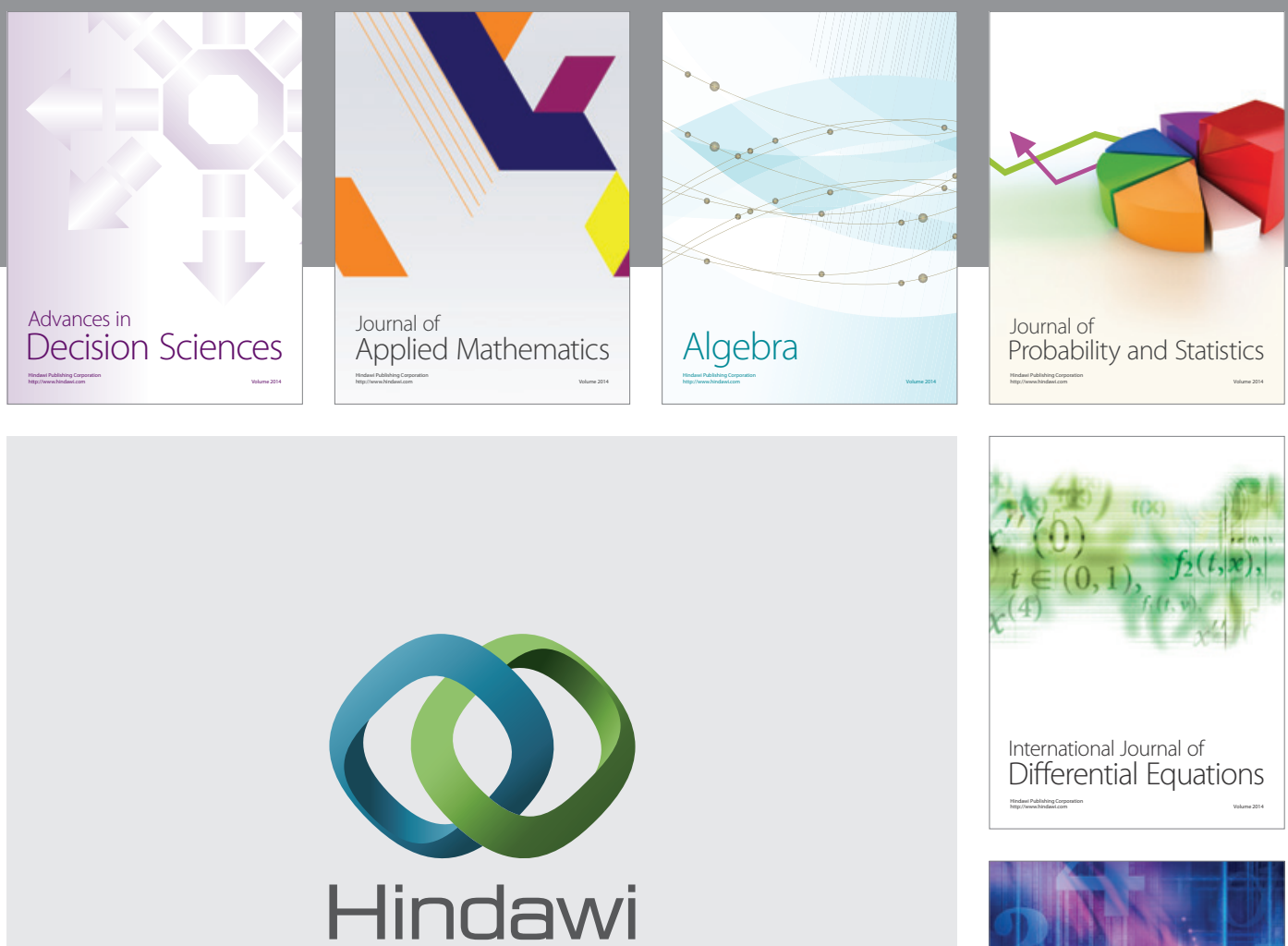

Submit your manuscripts at http://www.hindawi.com
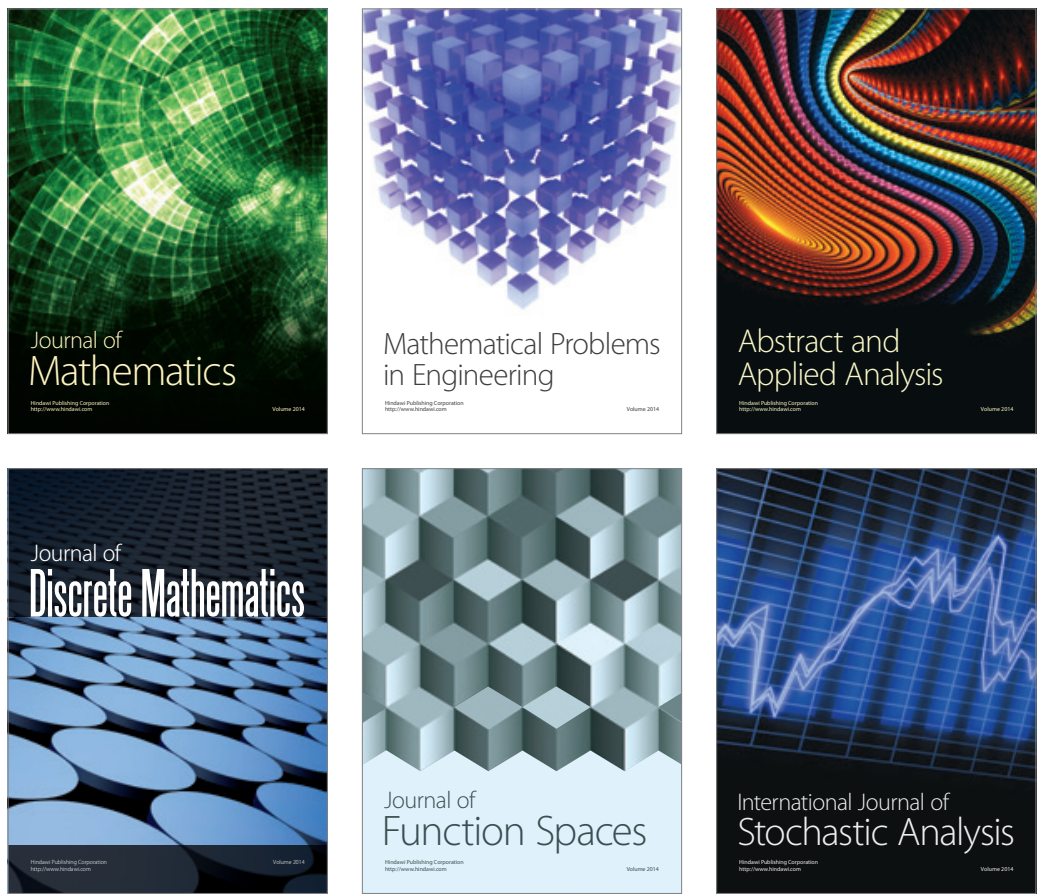

Journal of

Function Spaces

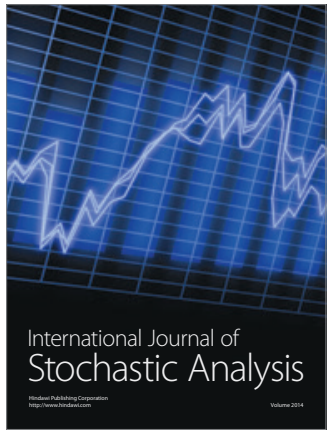

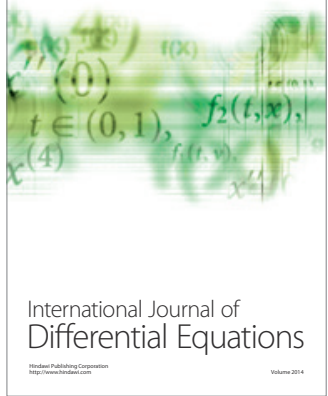
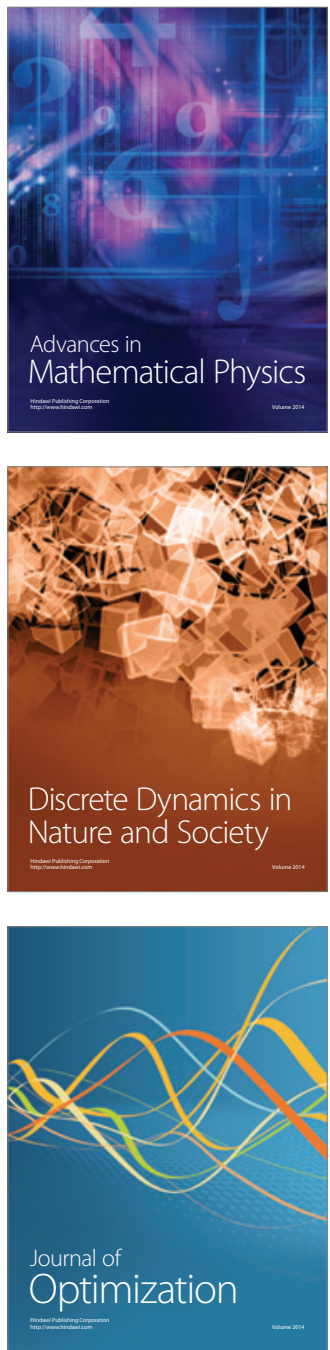